\title{
Modeling of Transient Two-Phase Flow in a Continuous Casting Mold Using Euler-Euler Large Eddy Simulation Scheme
}

\author{
Zhong-qiu LIU, ${ }^{1)}$ Bao-kuan $\mathrm{LI}^{1{ }^{1 / *}}$ Mao-fa JIANG ${ }^{1)}$ and Fumitaka TSUKIHASHI ${ }^{2)}$ \\ 1) School of Materials and Metallurgy, Northeastern University, Shenyang, 110819 China. \\ 2) Department of Advanced \\ Materials, Graduate School of Frontier Science, the University of Tokyo, 5-1-5 Kashiwanoha, Kashiwa, Chiba, 277-8561 Japan.
}

(Received on October 16, 2012; accepted on December 17, 2012)

\begin{abstract}
Euler-Euler Large Eddy Simulation (EELES) scheme has been developed to simulate the two-phase flow of argon gas and molten steel in slab continuous casting mold. The Euler-Euler approach is used to describe the equations of motion of the two-phase flow. The drag force, lift force and virtual mass force are incorporated in this model. Both turbulence of argon gas and molten steel are simulated using large eddy simulation (LES). Simulation results agree acceptably well with the water model experimental measurements of instantaneous flow structures. The flow pattern in the lower recirculation zone is expected to be asymmetrical between the left and right sides of the mold. The flow pattern is changeover; the direction of flow deviation is different from time to time. The time intervals for changeover appeared to vary randomly. The long-term asymmetry in the lower roll is due to the turbulent nature instead of the variation of other operating parameters. The turbulent flow in the mold includes multiple vortices. Those vortexes make the flow field to be more complex. Two typical transient flow structures, consisting of clockwise or counterclockwise rotational direction vortices, are found in the upper roll.
\end{abstract}

KEY WORDS: continuous casting mold; Euler-Euler large eddy simulation; two-phase flow; asymmetrical flow pattern.

\section{Introduction}

In the continuous casting process of steel industry, argon gas is injected into the nozzle to encourage mixing, to help prevent clogging, and to promote the flotation of solid inclusion particles from the molten steel. It usually enters the continuous casting mold after injection into the submerged entry nozzle (SEN), and a lot of large bubbles will be safely escape from the top surface, but some smaller bubbles (surrounded by inclusions) either be harmfully entrapped in the shell, at the solid/liquid interface of the solidifying metal, ${ }^{1-4)}$ causing pinhole defects, as shown in Fig. 1. Understanding the behavior of molten steel-argon gas two-phase jet and molten steel flow around the jet is essential for the design of effective methods of removing small bubbles.

Two approaches are mostly used to simulate the flow in continuous casting process: the Euler-Lagrange (E-L) and Euler-Euler (E-E) approach. Detailed descriptions of the E$\mathrm{L}$ and E-E models are found in previous studies. ${ }^{5,6)}$ The E$\mathrm{L}$ approach has been widely used to study the transport of inclusions and bubbles in continuous casting process. Thomas et al. . $^{7)}$ tracked the trajectories of individual bubbles through the molten steel in a mold using a Lagrangian approach for particle transport. The effect of the argon bubbles on the steel flow pattern was neglected, so the results only apply to low argon flow rates. Miki et al. ${ }^{1,8)}$ used the

* Corresponding author: E-mail: libk@smm.neu.edu.cn DOI: http://dx.doi.org/10.2355/isijinternational.53.484 dispersed phase model (DPM) to study the mechanism of bubbles entrapment and influence of gas bubbles on the steel flow, and the large eddy simulation was used to simulate the transient flow. Li et al. ${ }^{9,10)}$ employed a homogeneous fluid model with variable density to tackle the molten steelargon gas flow, considering the static magnetic-field application in the continuous casting process. Whereas, Thomas et $a l .{ }^{11)}$ used an E-E model to calculate the volume fraction and velocities of the argon bubble, and its effort on the molten steel flow. Creech ${ }^{12)}$ investigated the turbulent flow of molten steel and argon bubbles in the mold using the E-E

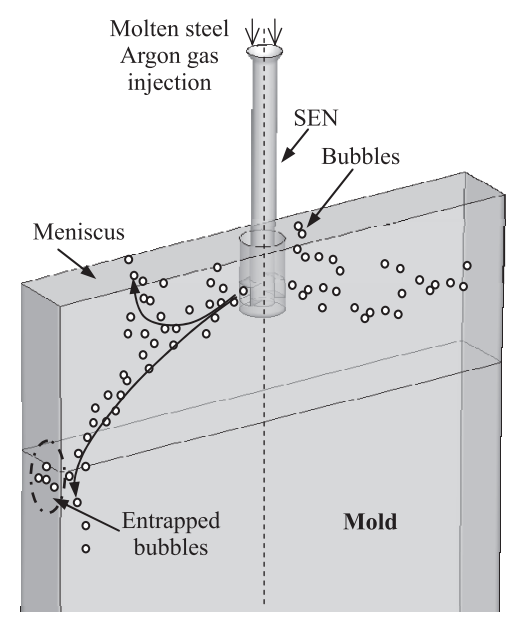

Fig. 1. Schematic of argon bubbles capture in the continuous casting mold. 
approach in the CFD program CFX, in which one velocity field for the molten steel and a separate velocity field for the gas phase are solved. The momentum equation for each phase is affected by the other phase through interphase drag terms. Bai et al. ${ }^{2,13)}$ used the same approach to simulate the time-averaged flow of argon bubbles in molten steel, considering only an empirical interphase drag force between molten steel and argon bubbles.

The asymmetrically distribution of defects in the slab has been found in recent flaw detection of slab. ${ }^{14)}$ However, most of the mathematical simulations reported in the literature provide time-averaged values of the turbulent flows; do not provide information about the transient flows and velocity fluctuations that are characteristic of turbulent flow phenomena. The large eddy simulation (LES) was successfully applied to obtain the asymmetrically flow of molten steel in the single phase in the mold. ${ }^{15-18)}$ But there is no work published on studying the two-phase flow for argon gas-molten steel in mold using the LES.

In the present work, the EELES model is firstly used to simulate the argon gas-molten steel flow in slab continuous casting process. The Eulerian approach will be used for the description of both argon gas and molten steel phases. The drag force, lift force and virtual mass force are incorporated in the model to consider the interaction between the dispersed gas phase (argon bubble) and the continuous liquid phase (molten steel). The turbulence of the two phases can be described using LES model.

\section{Description of Mold Structures}

The continuous casting process with the vertical-bending type machine has far fewer internal defects problems than the same process with the curved type caster. However, even with the vertical-bending caster, defects are still a problem in high quality steel product. ${ }^{1)}$ When the gas bubbles penetrated to the bending part of mold, it is different for them to rise to the top surface, where they dissipate into the atmosphere, but they would be captured by the solidified shell. In order to get the more real flow information, the continuous casting mold considered strand casters have a vertical part and a bending part, as exhibited in Fig. 2. In order to visualize the flow pattern and validate the mathematical model, a one-third-scale water model was established, including tundish, upper nozzle, slide gate, submerged entry nozzle (SEN) and casting mold. The diameter of the tiny horizontal pores used in the upper nozzle of water model is $1.0 \mathrm{~mm}$. There are 24 pores around the upper nozzle. The air was injected through the tiny horizontal pores into turbulent liquid flowing vertically down the wall, as shown in Fig. 3. The fluid properties and operating conditions used in water model and numerical simulation are given in Table $\mathbf{1 .}$

\section{Mathematical Model Formulation}

This calculation model is composed of the Euler and the LES approach. Inhomogeneous Euler-Euler approach is used to descript of both phases. The phases share this domain and interpenetrate as they move within it. Inhomogeneous multiphase flow refers to the case where separate velocity fields and other relevant fields exist for each fluid.

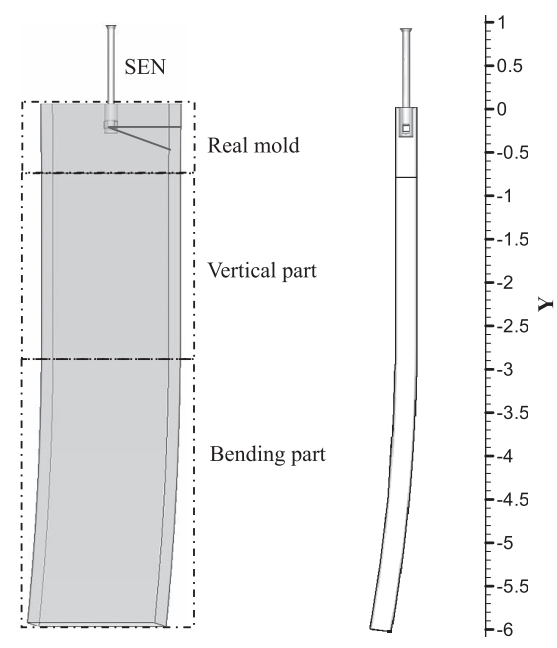

Fig. 2. Schematic of the calculation model.

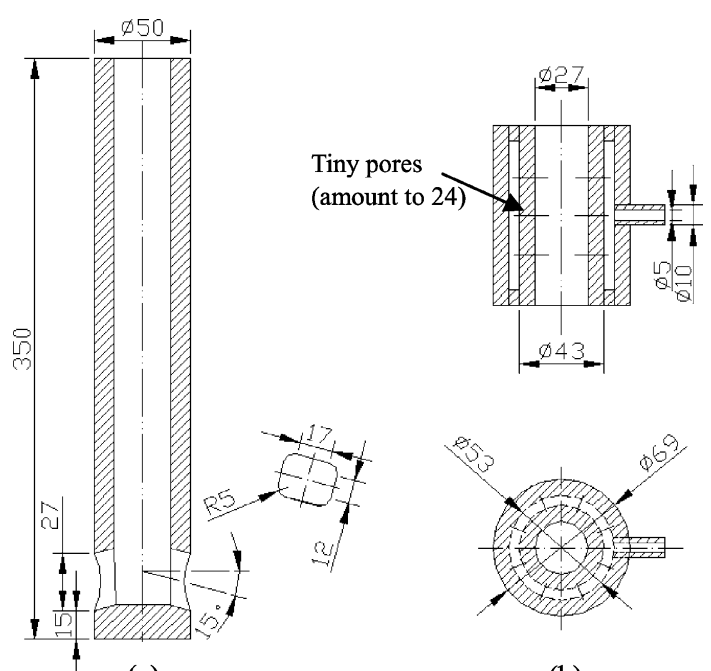

(a)

(b)

Fig. 3. Schematic of submerged entry nozzle (a) and upper nozzle (b).

The LES turbulence model is used to take into account the turbulence in the system. The sub-grid scale modeling is based on the Smagorinsky kernel. It should be note that the model shares the pressure field for both of fluids. The fluids interact via interphase transfer terms. Figure 4 shows the procedure details in the numerical calculation.

\subsection{Governing Equations}

The equations of motion for $k$ phase in an Euler-Euler simulation are generally given as follows: ${ }^{19,20)}$

$$
\begin{gathered}
\frac{\partial\left(\alpha_{k} \rho_{k}\right)}{\partial t}+\nabla \cdot\left(\alpha_{k} \rho_{k} u_{k}\right)=0 \ldots \ldots . . . \\
\frac{\partial\left(\alpha_{k} \rho_{k} u_{k}\right)}{\partial t}+\nabla \cdot\left(\alpha_{k} \rho_{k} u_{k} u_{k}\right) \\
=-\nabla \cdot\left(\alpha_{k} \tau_{k}\right)-\alpha_{k} \nabla P+\alpha_{k} \rho_{k} g^{\prime}+M_{I, k}
\end{gathered}
$$

The terms on the right-hand side of Eq. (2) are, respectively, representing the stress, the pressure gradient, gravity, and the momentum exchange between the phases due to interface force.

The stress term of $k$ phase is described as follows: 
Table 1. Comparison of process parameters between water model and actual continuous casting mold.

\begin{tabular}{|c|c|c|c|c|c|}
\hline Parameters & Water-Air System & Steel-Argon System & Parameters & Water-Air System & Steel-Argon System \\
\hline Diameter of SEN (mm) & 27 & 80 & Length of vertical part (mm) & 667 & 2000 \\
\hline Length of SEN (mm) & 300 & 900 & Length of bending part (mm) & 1067 & 3200 \\
\hline Exit down angle of nozzle $\left(^{\circ}\right)$ & 15 & 15 & Radius of curvature (mm) & 10250 & 10250 \\
\hline Height of SEN port (mm) & 27 & 80 & Fluid flow rate (L/min) & 45.44 & 710 \\
\hline Width of SEN port (mm) & 20 & 60 & Gas flow rate, cold (L/min) & 5 & 15.6 \\
\hline Submergence depth of SEN (mm) & 100 & 300 & Fluid density $\left(\mathrm{kg} / \mathrm{m}^{3}\right)$ & 1000 & 7020 \\
\hline Width of mold (mm) & 579 & 1736 & Gas density $\left(\mathrm{kg} / \mathrm{m}^{3}\right)$ & 1.29 & 0.56 \\
\hline Thickness of mold (mm) & 76 & 228 & Fluid viscosity $\left(\mathrm{kg} \cdot \mathrm{m}^{-1} \cdot \mathrm{s}^{-1}\right)$ & 0.001 & 0.0056 \\
\hline Length of real mold (mm) & 300 & 900 & Gas viscosity $\left(\mathrm{kg} \cdot \mathrm{m}^{-1} \cdot \mathrm{s}^{-1}\right)$ & $1.7 \times 10^{-5}$ & $7.42 \times 10^{-5}$ \\
\hline
\end{tabular}

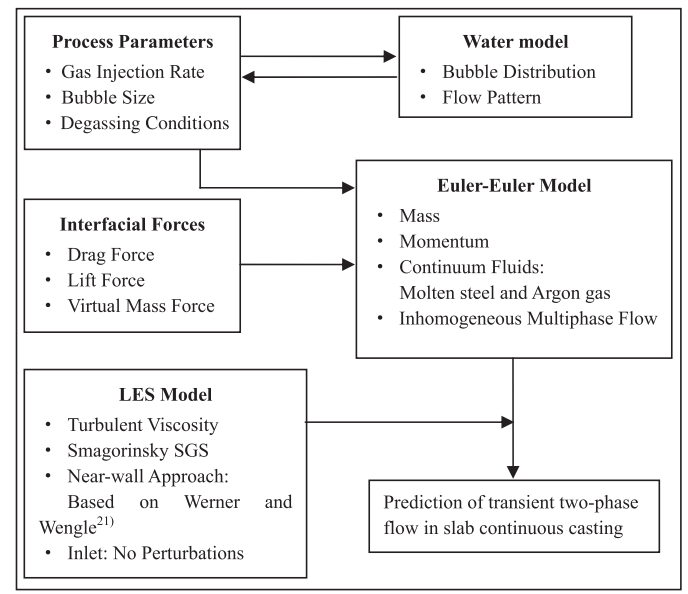

Fig. 4. Flow chart of model solution methodology of EELES.

$$
\tau_{k}=-\mu_{e f f, k}\left(\nabla u_{k}+\left(\nabla u_{k}\right)^{T}-\frac{2}{3} \mathrm{I}\left(\nabla \cdot u_{k}\right)\right) \ldots
$$

where $\mu_{e f f, k}$ is the effective viscosity. The effective viscosity of the liquid phase is composed of three contributions: the molecular viscosity, the turbulent viscosity and an extra term due to bubble induced turbulence.

$$
\mu_{e f f, l}=\mu_{L, l}+\mu_{T, l}+\mu_{B I, l}
$$

The calculation of the effective gas viscosity is based on the effective liquid viscosity as was proposed by Jakobsen et al. ${ }^{21)}$

$$
\mu_{e f f, g}=\frac{\rho_{g}}{\rho_{l}} \mu_{e f f, l}
$$

The model proposed by Sato \& Sekiguchi ${ }^{22)}$ has been used to take account of the turbulence induced by the movement of the bubbles. The expression is:

$$
\mu_{B I, l}=\rho_{l} C_{\mu, B I} \alpha_{g} d_{g}\left|u_{g}-u_{l}\right|
$$

with a model constant $C_{\mu, B I}$, which is equal to 0.6.

\subsubsection{Large Eddy Simulation}

Turbulent flows are characterized by eddies with a wide range of length and time scales. The largest eddies are typically comparable in size to the characteristic length of the mean flow. The smallest scales are responsible for the dissipation of turbulence kinetic energy.

The velocities in Eqs. (1) and (2) are defined as follows:

$$
u_{k}=\tilde{u}_{k}-u_{k}^{\prime}
$$

where $u_{k}$ is the part of the velocity for $k$ phase that will be resolved in the numerical simulations, $\tilde{u}_{k}$ is the instantaneous velocity and $u_{k}^{\prime}$ is the unresolved part of the numerical simulation. Most models derive Eqs. (1) and (2) through ensemble averaging, $\tilde{u}_{k}$ and $u_{k}^{\prime}$ represent the mean velocity and the fluctuating velocity. When Eqs. (1) and (2) are obtained through a filtering operation; these terms are, respectively, the grid scale and the sub-grid scale (SGS) velocities. In LES, large eddies are resolved directly, while small eddies are modeled.

The key element in LES is the sub-grid scale model, which determines the effect of the unresolved turbulent scales. The model proposed by Smagorinsky ${ }^{23)}$ is used to calculate the turbulent viscosity $\mu_{T, l}$.

$$
\mu_{T, l}=\rho_{l}\left(C_{S} \Delta\right)^{2}|S|
$$

where $C_{S}$ is the model constant with a value of $0.1 . S$ is the characteristic filtered rate of strain and $\Delta=\left(\Delta_{i} \Delta_{j} \Delta_{k}\right)^{1 / 3}$ is the filter width.

\subsubsection{LES Near-wall Treatment}

There is an alternative near-wall approach based on the work of Werner and Wengle, ${ }^{24)}$ who proposed an analytical integration of the power-law near-wall velocity distribution resulting in the following expressions for the wall shear stress:

$$
\begin{aligned}
& \left|\tau_{\omega}\right|= \\
& \begin{cases}\frac{2 \mu_{e f f}\left|u_{p}\right|}{\Delta_{w}} & \text { for }\left|u_{p}\right| \leq \frac{\mu_{e f f}}{2 \rho \Delta} A^{\frac{2}{1-B}} \\
\rho\left[\frac{1-B}{2} A^{\frac{1+B}{1-B}}\left(\frac{\mu_{e f f}}{\rho \Delta_{w}}\right)^{1+B}+\frac{1+B}{A}\left(\frac{\mu_{e f f}}{\rho \Delta_{w}}\right)^{B}\left|u_{p}\right|\right]^{\frac{2}{1+B}} \text { for }\left|u_{p}\right|>\frac{\mu_{e f f}}{2 \rho \Delta} A^{\frac{2}{1-B}}\end{cases}
\end{aligned}
$$

where $u_{p}$ is the wall-parallel velocity, $A=8.3, B=1 / 7$ are the constants based on the work of Werner and Wengle, and $\Delta_{w}$ is the near-wall control volume length scale.

\subsubsection{Interfacial Forces}

The momentum exchange term $M_{I, k}$ in Eq. (2) describing the interface forces is given as follows: 


$$
M_{I, l}=-M_{I, g}=M_{D, l}+M_{L, l}+M_{V M, l}
$$

where the terms on the right hand side of Eq. (10) are respectively forces due to drag, lift and virtual mass force, respectively. For an extensive discussion of these forces the reader is referred to the works of Deen et al. ${ }^{19)}$ and Dhotre et al. $^{20)}$

The drag force is given as follows:

$$
M_{D, l}=-\frac{3}{4} \alpha_{g} \rho_{l} \frac{C_{D}}{d_{g}}\left|u_{g}-u_{l}\right|\left(u_{g}-u_{l}\right)
$$

For particle Reynolds numbers, $\operatorname{Re}_{\mathrm{lg}}=\rho_{l}\left|u_{g}-u_{l}\right| d_{g} / \mu_{L}$, that are sufficiently large for inertial effects to dominate viscous efforts(the inertial or Newton's regime), the drag coefficient becomes independent of Reynolds number:

$$
C_{D}=0.44, \quad 1000 \leq \operatorname{Re} \leq 2 \times 10^{5}
$$

A bubble traveling through a fluid in shearing motion will experience lift force transverse to the direction of motion. The effort of shearing motion in the liquid phase on the movement of the gas phase is modeled through the lift force

$$
M_{L, l}=\alpha_{g} \rho_{l} C_{L}\left(u_{g}-u_{l}\right) \times \nabla \times u_{l}
$$

where $C_{L}$ is a model constant. The present work concentrates on bubbly flow regime with $2.88 \mathrm{~mm}$ small spherical bubbles, where only positive lift force coefficient is sufficient which can be in the range of 0.1 to 0.5 . Drew and Lahey ${ }^{25)}$ proposed $C_{L}=0.5$ based on objectivity arguments. So $C_{L}$ is set to 0.5 .

The virtual mass force accounts for relative acceleration, the additional work performed by the bubbles in accelerating the liquid surrounding the bubble. The acceleration of the liquid is taken into account through the virtual mass force, which is given by

$$
M_{V M, l}=\alpha_{g} \rho_{l} C_{V M}\left(\frac{D u_{g}}{D t}-\frac{D u_{l}}{D t}\right)
$$

where the virtual mass coefficient $C_{V M}$ is taken to be 0.5 for individual spherical bubbles. The $D / D t$ operators denote the substantial derivatives in the two phases.

\subsection{Method of Solution}

An inhomogeneous (inter-fluid transfer) model was used for Euler-Euler multiphase flow. Each fluid possesses its own flow field and the fluids interact via interphase transfer terms. For the LES, a conventional Smagorinsky sub-grid model was adapted to modeling the turbulence of the twophase. The coupled EELES equations were discretized using the finite-volume approach on a structured Cartesian staggered grid.

\subsubsection{Grid Requirement}

The mesh and time step are an inherent part of the model. LES models make use of the grid scale for filtering out the turbulence. All scales smaller than the resolution of the mesh is modeled and all scales larger than the cells are computed. Therefore, In LES is to resolve as much as possible, and to have as fine a grid as feasible for the available computer hardware. On other hand, this requirement conflicts with the fact that the computational grid should be large enough compared to the bubble diameter in order to provide significant statistical samples. In the present work, the EulerEuler approach for multiphase modeling with LES has been considered, and therefore has to consider the resolution requirements of both techniques in order to choose a satisfactory grid. For EELES, Dhotre et al. ${ }^{20)}$ indicated that the control volume size is large enough to encompass all the interface details. This was the intrinsic assumption in the derivation of the Euler-Euler model equations, and strictly has to be satisfied at the discrete level as well. They observed that relative coarse grids (ratio $\Delta / \mathrm{d}_{\mathrm{g}}$ is 1.2 for the fine grid and 2.5 for the coarse grid) can be used without losing any fundamental characteristics of the flow.

In this study, we used grid size, which is larger than bubble diameter; the bubble diameter is specified according to the water model test $(2.88 \mathrm{~mm})$. This computational domain was discretized with a structured Cartesian grid consisting of 1.8 million cells. The time step of the simulation was 0.0005 seconds.

\subsubsection{Numerical Details}

A uniform velocity for two phases is prescribed at the inlet opening based on the casting speed, and "No Perturbations" option inlet boundary conditions for the LES model. The top surface of the mold cavity is modeled as degassing boundary conditions, which dispersed argon bubbles are permitted to escape, but the molten steel is not. A constant pressure boundary condition is used at the outlet ports in the bottom wall, where the gradients of all the other variables are set to zero. The no-slip boundary condition is employed at the wall boundaries.

Argon gas is injected into the SEN at a room temperature. It expands descending in the nozzle due to heat transfer. Thus, the argon gas injection flow rate used in the numerical model is the hot argon flow rate. Gas injection may be characterized by the average gas volume fraction $\left(\overline{f_{g}}\right)$, which can be found from ${ }^{26)}$

$$
\overline{f_{g}}=\frac{\beta Q_{g}}{\beta Q_{g}+Q_{l}}
$$

where $Q_{g}$ is the gas injection flow rate at STP (standard temperature of $25^{\circ} \mathrm{C}$ and pressure of $1 \mathrm{~atm}$ ) and $Q_{l}$ is the liquid flow rate through the nozzle inlet, found by multiplying the casting speed by the cross-sectional area of the strand. $\beta$ is the factor of the gas volume expansion due to the temperature and pressure change, which is about $5 .^{11)}$

In addition, LES has to be run for a sufficiently long flowtime to obtain stable statistics of the flow being modeled. As a result, the computational cost involved with LES is normally orders of magnitudes higher than that for steady RANS calculations in terms of memory (RAM) and CPU time. So this paper starts by running a steady state flow simulation using a Reynolds-averaged turbulence model, standard $\mathrm{k}-\varepsilon$. Run until the flow field is reasonably converged and then run LES until the flow becomes statistically steady. The best way to see if the flow is fully developed and statistically steady is to monitor solution variables (e.g., velocity components) at selected locations in the flow. Additionally, it will help in reducing the time needed for the LES simulation to reach a statistically stable mode.

A second-order-accurate central difference scheme was used for the discretization of the convection terms. The dis- 
crete approximation of the transient term for the time integration was handled through the second order backward Euler scheme. The SIMPLE procedure was used for the velocity-pressure coupling. The time step of the simulation was 0.0005 seconds. The flow was simulated for $200 \mathrm{~s}$ and the data was monitored in the process of calculation.

\section{Model Validation}

\subsection{Bubble Distribution}

After fluid and bubble flow reached a steady state in water model experiment, the picture of bubble dispersion were taken using a high speed camera with 4500 frames per second. The photograph of water-air two-phase jet is shown in Fig. 5(a), which is obtained at a given water flow rate of $45.44 \mathrm{~L} / \mathrm{min}$ and gas flow rate of $5 \mathrm{~L} / \mathrm{min}$. The white patches in the figure represent bubbles. Most of bubbles tend to float toward the top surface due to buoyancy acting on air bubbles and are subsequently removed from the mold. Large bubbles rise toward the top surface quickly, while smaller bubbles are carried deep into the mold, and the penetrating length of the smaller bubbles is lengthened. Through the image analyzing method, the sizes of bubbles were measured. The measured mean air bubble diameter in water model is $2.88 \mathrm{~mm}$, as shown in Fig. 5(b), which is approximately within the range of argon bubble in real continuous casting molds. The mean bubble size data from water model results was used as the initial mean diameter in the current mathematical model. The gas fraction distribution results are given in Fig. 5(c). It can be seen that the distributions and penetrating length of argon gas agree well with the

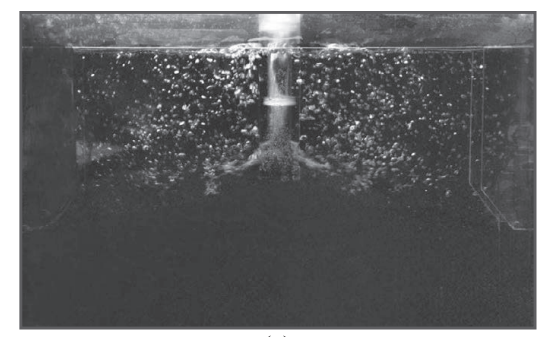

(a)

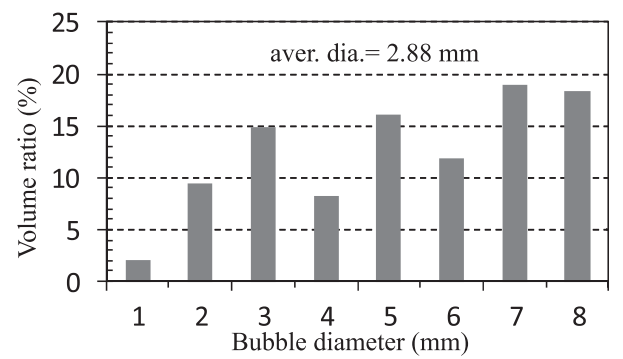

(b)
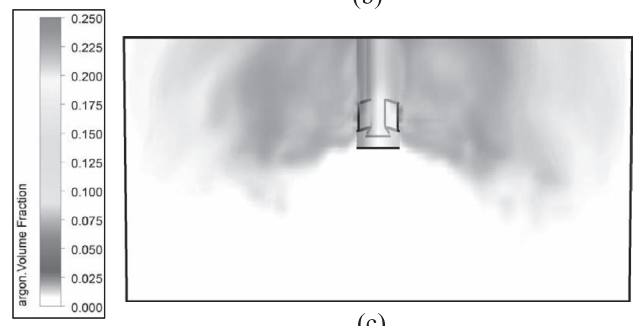

(c)

Fig. 5. Distribution and size of bubbles from water model and EELES model. water model results. Most of the bubbles are crowded together in the upper region. After entering the mold with the liquid jet, the bubbles float quickly upward through the recirculation zone, and leave the top surface. Fewer and fewer bubbles stay in the jet as it travels across the mold. This agreement is remarkable, considering the crude assumptions regarding bubble shape and the lack of calibration parameters in the model.

\subsection{Asymmetric Flow Pattern}

Argon bubbles accumulated in the SEN are shear strained and broken down, forming smaller bubbles, and together with the molten steel entraining the mold. The long-term research of molten steel flow in the continuous casting mold shows that the bubbles can't be removed by the slag layer if their ascent rate is no faster than casting speed. They would flow together with the molten steel, and finally stranded in slab to form slab defects. The bubble distribution in casting slabs was investigated by the ultrasonic testing (UT) method at the three cross sections of slab, as shown in Fig. 6. The UT results revealed that bubble defects (many $\mathrm{Al}_{2} \mathrm{O}_{3}$ adhering to the surface of a bubble) present asymmetric distribution. And they were mainly found at the $1 / 4$ width of the slab.

Asymmetrical variations in the transient flow structure in the mold are important because they may be linked with observations of inclusion entrapment from side to side. They also may induce the serious top slag fluctuation, and the jumping of fish flow. Fluid flow and transport phenomena in casting molds are of a dynamic nature. Due to the fluctuating nature of turbulent flow, the asymmetric velocity field also provides asymmetric tracer dispersion patterns. But in most Reynolds-averaged simulations, symmetry is assumed between the flows in the two halves of the liquid pool. This assumption has been shown to be valid for longterm averages. However, transient flow in the two halves is different, as shown in Fig. 7. Gupta et al. ${ }^{27,28)}$ have found that the discharge of fluid at the mold exit was mostly asymmetrical, and the flow pattern was not stationary but changed over frequently. Unfortunately, there is yet no clear model for simulating the asymmetry two phase flow phenomena to occur in actual processes.

Figure 7 shows the fluid flow pattern inside the water model and the actual mold (the calculation model). This photograph was obtained by black-colored dye injection against a white background, as shown in Fig. 7(a). It can be

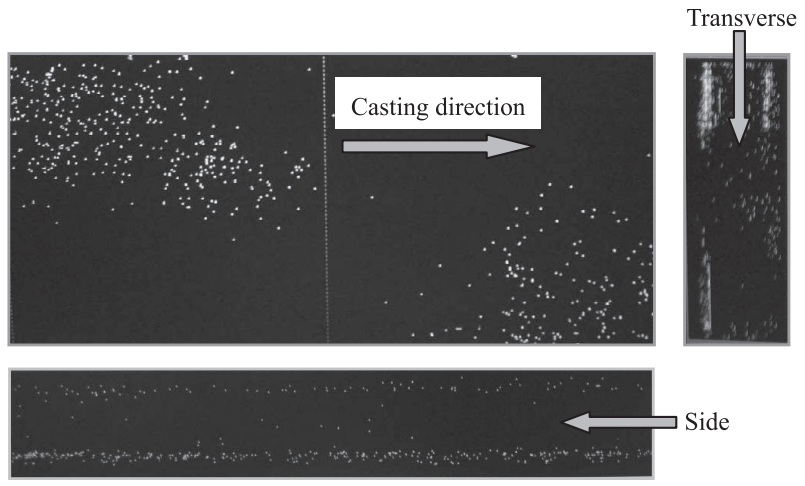

Fig. 6. Inclusion entrapment positions in slab billet. 


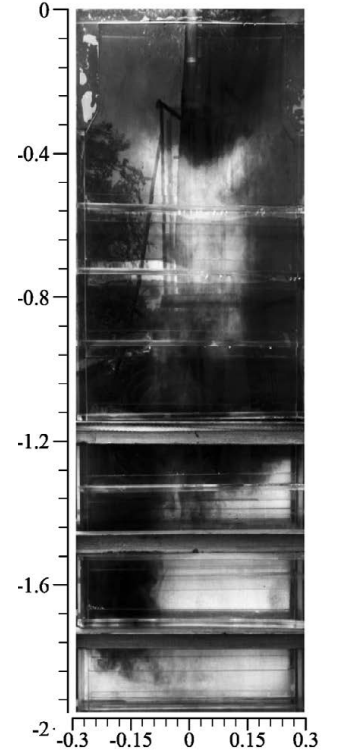

(a)
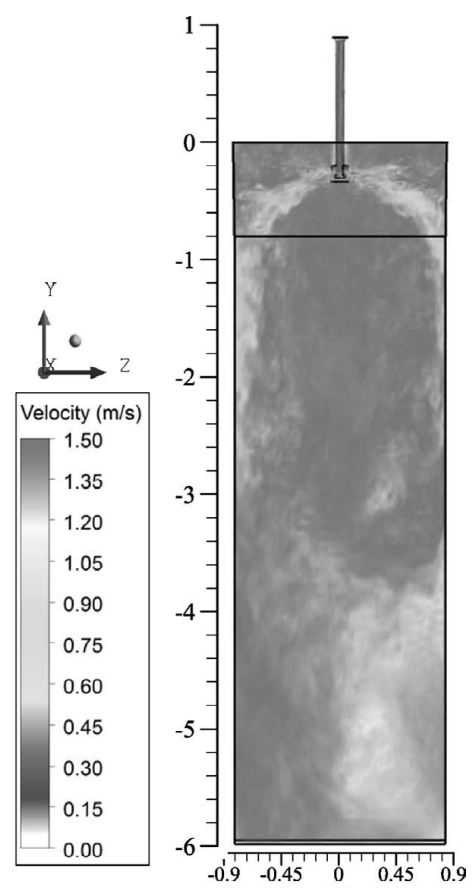

(b)
Fig. 7. Fluid flow pattern obtain from the water model experiment (a) and molten steel velocity contour obtain from EELES model (b).

noticed that the fluid jet flow is not symmetrical about the central plane. The jet flow on the left half descends in the downward direction, leading the fluid discharge through the left half of the mold. On the right half, there is a big colored domain. With this mathematical model, the flow pattern of molten steel inside the mold is given in Fig. 7(b). The flow pattern in the experiment and that predicted by the mathematical model are qualitatively very similar. The location of lower recirculation region eye was approximately the same in the two cases, which is at the interface of vertical part and bending part of the mold. The long-term asymmetry in the lower roll is due to the turbulent nature instead of the variation of other operating parameters. Overall, the EELES model predictions match well with the water model results for asymmetry flow.

\section{Transient Flow Structure}

Flow transients influence the transport of inclusion particles and bubbles carried by the jets into the liquid pool and might be the cause for intermittent and asymmetrical defects observed in plants. To save the steel and lower costs, it is necessary to control the steel cleanliness at the transient stages. However, even under the present circumstances, the LES approach proved to be very useful to complement the experimental finding of this work.

A typical instantaneous velocity field for a casting rate of $1.8 \mathrm{~m} / \mathrm{min}$ in the liquid pool is shown in Fig. 8. Molten steel (contain many argon bubbles) emerges from the inlet port as a jet, diffuses as it traverses across the liquid pool, impinges on the narrow face, and splits into two recirculation zones consisting of complex structures. The figure shows that the liquid pool is consist of multiple vortices. In addition, those vortexes make the flow field in the mold to be more complex. Figures 9(a) and 9(b) show the closer view of the

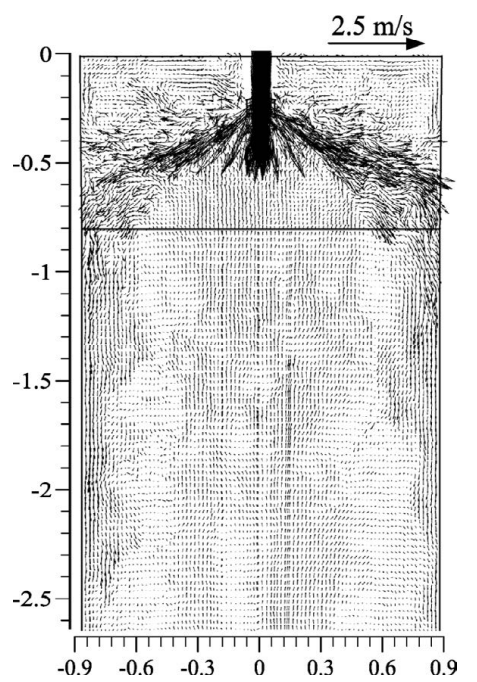

Fig. 8. Typical instantaneous velocity vector plot in mid-plane of the mold $(\mathrm{t}=150 \mathrm{~s})$.

velocity structures in the upper roll at an arbitrary time of 175 and 195 seconds, respectively. At the first time ( 175 seconds), the jet angle in the left side of the mold is close to the horizontal, to forming an intense upper recirculation flow, whereas the right-side jet hits the narrow face and splits partly upward toward the surface and partly downward. Because the jets enter the mold with strong turbulence, a randomly high jet tends to be deflected upward, whereas a randomly down jet tends to be deflected downward. And the right-side jet rotates in a counterclockwise direction, as seen from the right side of the mold. But $20 \mathrm{~s}$ later (195 s), the jet rotational direction change to clockwise. This suggests that the vortex size and rotational direction change with time variation.

The three velocity fluctuation components at the mold mid-plane at two pairs of monitoring points, symmetrically located at meniscus and $336 \mathrm{~mm}$ below the meniscus respectively, midway from the SEN and the narrow face are shown in Fig. 10. The pair of points (336 mm below meniscus) is located within the jets flow. The transient values were collected every 0.005 second for 200 seconds. The velocity distributions at various times show that the flow pattern is ever-changing. The difference between the rightand left-hand points is significant. The velocity fluctuations are seen to be large, with a magnitude comparable to the local mean velocities. Such an upward shift in the peak position ( $v$ velocity) is due to the buoyancy force acting on bubbles. This velocity variation is important, because the molten steel level fluctuations accompanying it are a major cause of defects in the process. Evidently, peak velocities in these cycles bring about sudden changes of shear stresses promoting radical changes of the flow pattern. This result fully shows that the molten steel turbulent field in the mold is transient and random. So the flow pattern of molten steel is changeover, but the time intervals for changeover appeared to vary randomly.

The velocity on the top surface of the mold plays an important role in the entrainment of slag. Shear flow and swirl flow at the interface of molten steel and mold flux lead to the slag entrainment. It is observed that the characteristic of large sudden "jumps" was seen in the water model exper- 

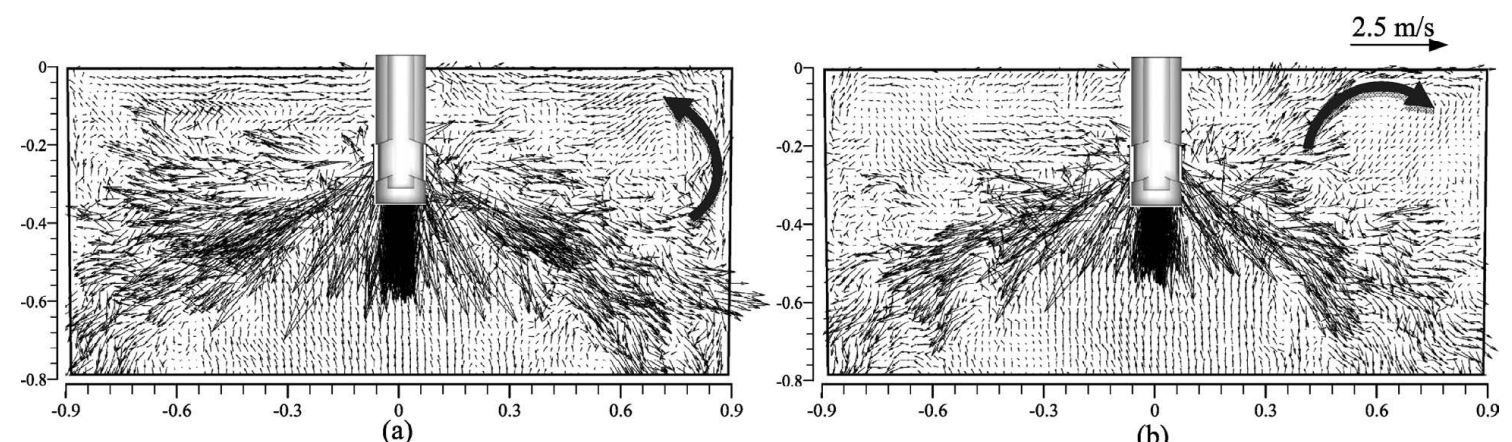

Fig. 9. Predicted flow patterns in the upper recirculation zone (a) $175 \mathrm{~s}$, counterclockwise (b) $195 \mathrm{~s}$, clockwise.
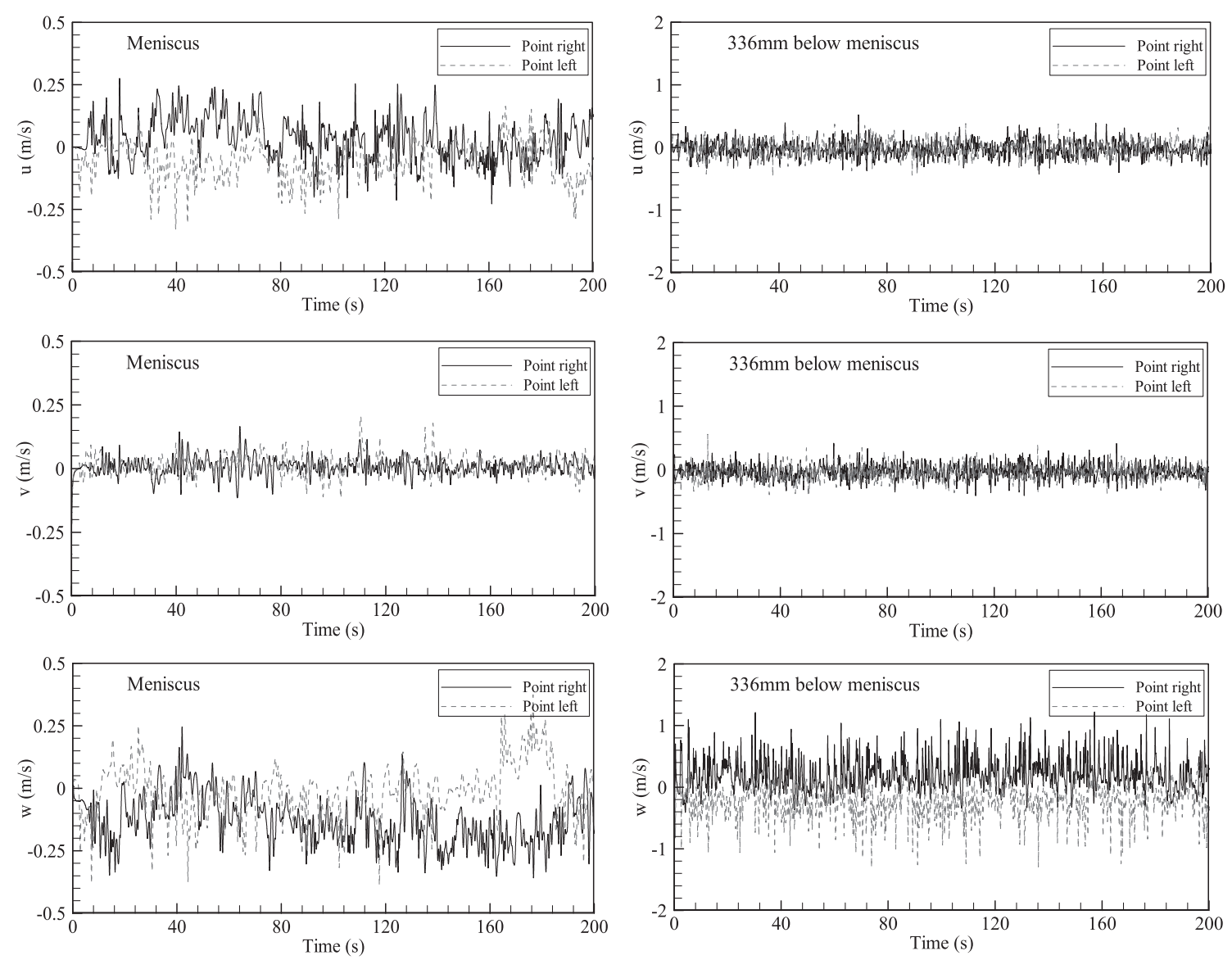

(a)

(b)

Fig. 10. Three components of instantaneous velocity fluctuations at mid-plane in different cases (a) meniscus (b) $336 \mathrm{~mm}$ below meniscus.

iment, as shown in Fig. 11(a). It can be seen that there are a lot of large bubbles. So this phenomenon is caused by the coalescence of many smaller bubbles. Industrial observation of the top surface revealed that there are several "exposed eyes" at the top surface of the real mold, around the SEN, where is the final escape location of bubbles on the top surface, as shown in Fig. 11(b), when the argon flow rate at STP is $15.6 \mathrm{~L} / \mathrm{min}$. Figure 12 shows the molten steel vertical velocity distribution along the center line of the meniscus at different times. The existence of positive and negative velocity value shows that the fluctuations are significant. The velocity component is mostly positive near the SEN, indicating that the flow is mostly upward with occasional downward excursions. Sharp velocity spikes are sometimes observed, and the positions are different at different times, such as point (1) to point (4). The mold fluxes in these positions have a big opportunity to be pushed away and may generate an "exposed eye" of the molten steel, as shown in Fig. 11, indicating serious reoxidation from the air and worsening slab quality.

The predicted gas volume fraction distribution in the upper mold is given in Fig. 13, obtained from the EELES model. Because of the strong buoyancy force, which is proportional to the density difference between liquid and gas, most of the gas bubbles float upward and escape from the top surface during the time that the liquid jet takes to travel from the SEN port to the narrow face wall. The escape region of many bubbles is close to the SEN, they accumulate at that location, which will induce serious surface fluctuation. 


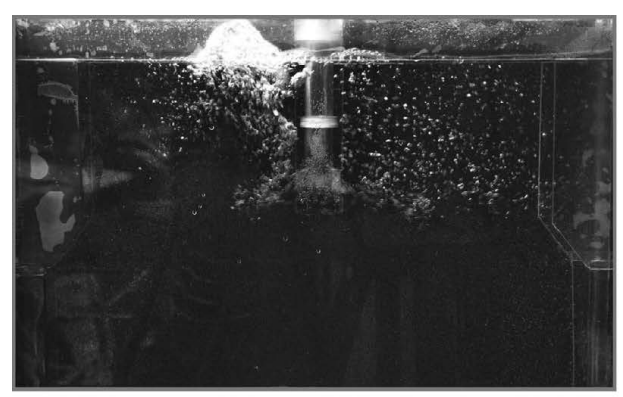

(a)

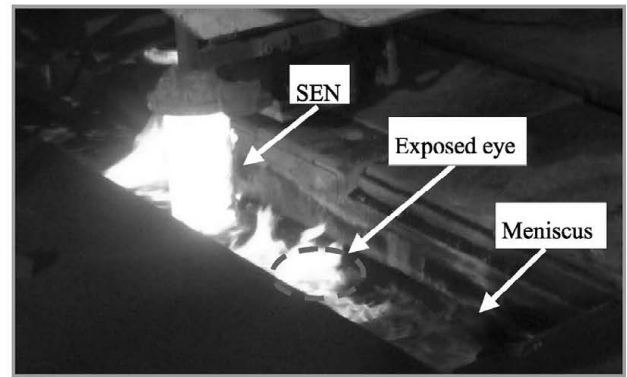

(b)

Fig. 11. Sudden "jumps" phenomenon from water model experiment (a) and industrial observation (b) at the top surface of mold.

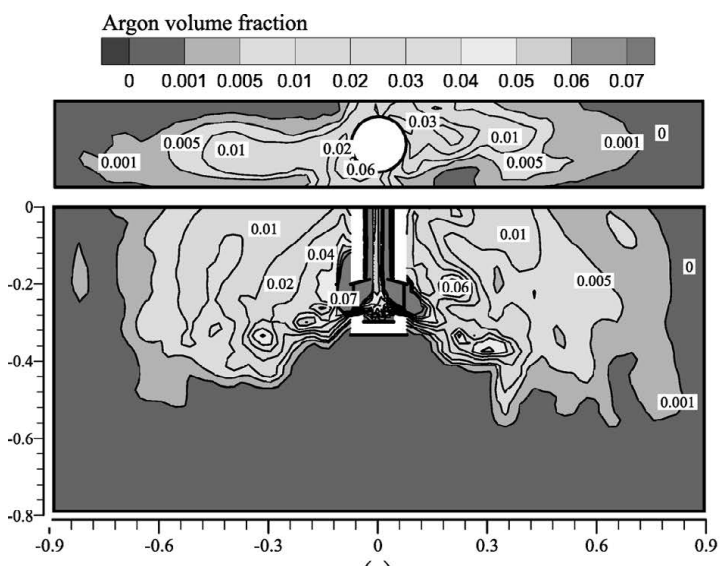

(a)

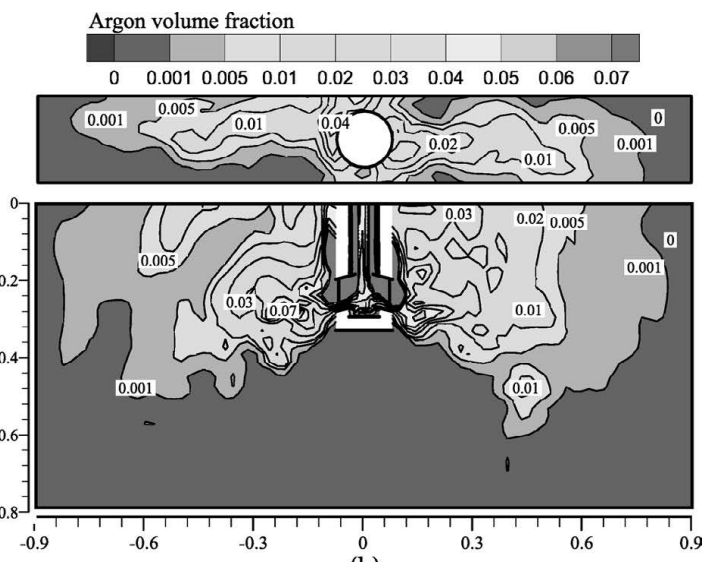

(b)

Fig. 13. Argon volume fraction distribution at center plane and meniscus in different cases (a) $175 \mathrm{~s} \mathrm{(b)} 195 \mathrm{~s}$.

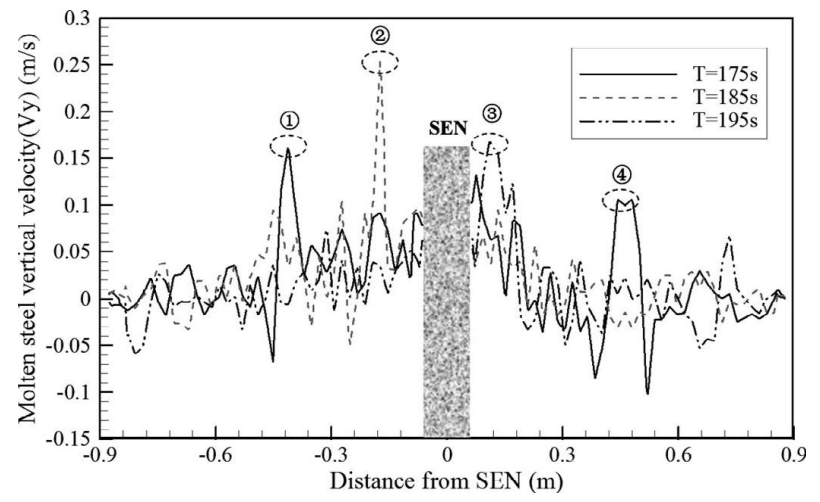

Fig. 12. Molten steel vertical velocity (Vy) along the center line of the meniscus.

Figure 14 illustrates the time history of argon volume fraction variations at the same points, which shows the similar variations to those in Fig. 10. The signals at the two points show a mean value of $\sim 0.01$, but with significant fluctuations. And sometimes the peak value is reach up to 0.03 . Long-term asymmetries of argon gas distribution are also observed between these two pairs of points in the jets.

These observations suggest that (1) the two-phase turbulent flow field is transient and random, (2) the flow pattern in the mold is expected to be asymmetrical, and (3) the longterm asymmetry in the lower roll is due to the turbulent nature instead of the variation of other operating parameters. Based on the above comparison of water model and predicted results, it is clear that the EELES model is able to predict

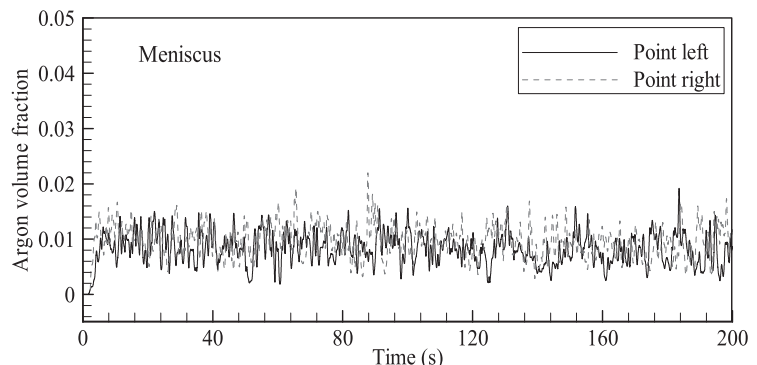

(a)

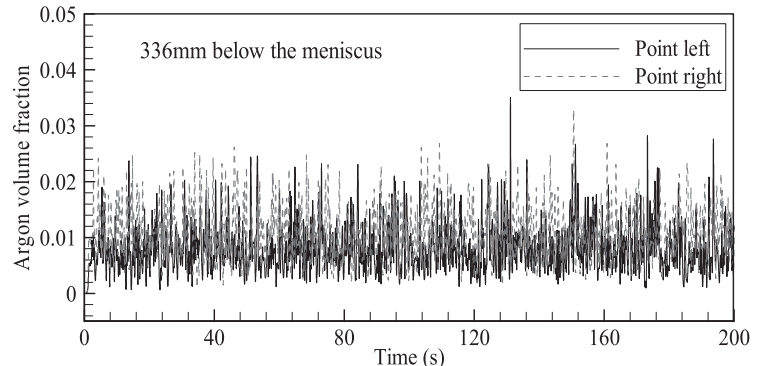

(b)

Fig. 14. Argon volume fraction variation at mid-plane in different cases (a) meniscus (b) $336 \mathrm{~mm}$ below meniscus.

the transient two-phase flow behavior in a continuous casting mold with reasonable success.

\section{Conclusions}

The EELES model developed in the present study has been carried out for the two-phase flow of argon gas and 
molten steel in a continuous casting mold. Theoretical, UT testing, and water model experimental instantaneous velocity fields have been compared. The following conclusions are concluded from this work.

(1) The EELES approach is able to predict qualitatively the instantaneous velocity fields of the two-phase flow in the mold.

(2) Large bubbles rise toward the top surface quickly, while smaller bubbles are carried deep into the mold, and the penetrating length of the smaller bubbles is lengthened. The measured mean bubble size with water model is $2.88 \mathrm{~mm}$ at the given flow rate.

(3) The flow pattern in both water model and this CFD model are expected to be asymmetrical. The location of lower recirculation region eye was approximately at the interface of vertical part and bending part of the mold. The two-phase turbulent flow field is transient and random, the direction of flow deviation is different from time to time. The time intervals for changeover appeared to vary randomly. The longterm asymmetry in the lower roll is due to the turbulent nature instead of the variation of other operating parameters.

(4) The turbulent flow in the mold includes multiple vortices. Those vortices make the flow field to be more complex. Two typical transient flow structures, consisting of clockwise or counterclockwise rotational direction vortices, are found in the upper roll.

(5) The final escape location of bubbles is around the SEN. Characteristic of large sudden "jumps" was also seen in the water model experiment. The mold flux in this region has a big opportunity to be pushed away and may generate an "exposed eye" of the molten steel, indicating serious reoxidation from the air and worsening slab quality.

\section{Acknowledgements}

Authors are grateful to the National Natural Science Foundation of China and Baosteel Co., Ltd. for support of this research, Grant No. 50934008.

\section{Nomenclature}

$A$ : Model constant, dimensionless

$B$ : Model constant, dimensionless

$C_{D}$ : Drag coefficient, dimensionless

$C_{L}$ : Lift force model constant, dimensionless

$C_{S}$ : Sub-grid scale model constant, dimensionless

$C_{V M}$ : Virtual mass force model constant, dimensionless

$C_{\mu, B I}$ : Sato \& Sekiguchi model constant, dimensionless

$d_{g}$ : Bubble diameter [m]

$\overline{f_{g}}$ : Average gas volume fraction, dimensionless

$g^{\prime}:$ Acceleration of gravity $\left[\mathrm{m} / \mathrm{s}^{2}\right]$

$g$ : Gas phase, dimensionless

I: Unity tensor, dimensionless

$k$ : $k$ phase, contain the liquid phase and gas phase, dimensionless

$l$ : Liquid phase, dimensionless

$M_{D}$ : Drag force $\left[\mathrm{N} / \mathrm{m}^{3}\right]$

$M_{I}$ : Total interfacial force $\left[\mathrm{N} / \mathrm{m}^{3}\right]$

$M_{L}:$ Lift force $\left[\mathrm{N} / \mathrm{m}^{3}\right]$

$M_{V M}:$ Virtual mass force $\left[\mathrm{N} / \mathrm{m}^{3}\right]$
$P: \quad$ Static pressure $\left[\mathrm{N} / \mathrm{m}^{2}\right]$

$Q:$ Fluid flow rate $\left[\mathrm{m}^{3} / \mathrm{s}\right]$

$\mathrm{Re}_{\mathrm{lg}}$ : Bubble Reynolds numbers, dimensionless

$S$ : Characteristic filtered rate of strain $[1 / \mathrm{s}]$

$t$ : Time [s]

$u_{k}$ : Fluid velocity of $k$ phase $[\mathrm{m} / \mathrm{s}$ ]

$\tilde{u}_{k}:$ Instantaneous velocity $[\mathrm{m} / \mathrm{s}]$

$u_{k}^{\prime}$ : Mean or grid scale velocity $[\mathrm{m} / \mathrm{s}]$

$u_{p}$ : Wall-parallel velocity $[\mathrm{m} / \mathrm{s}]$

$\alpha_{k}$ : Volume fraction of $k$ phase, dimensionless

$\alpha_{g}$ : Volume fraction of gas phase, dimensionless

$\beta$ : Gas volume expansion constant, dimensionless

$\rho_{k}$ : Fluid density of $k$ phase $\left[\mathrm{kg} \cdot \mathrm{m}^{-1} \cdot \mathrm{s}^{-2}\right]$

$\mu_{\text {eff }, k}: \quad$ Effective viscosity of $k$ phase [Pa's]

$\mu_{T:}$ Turbulent viscosity $\left[\mathrm{m}^{2} / \mathrm{s}\right]$

$\mu_{B I}$ : Turbulent viscosity of bubble induced $\left[\mathrm{m}^{2} / \mathrm{s}\right]$

$\mu_{L}:$ Molecular viscosity $\left[\mathrm{m}^{2} / \mathrm{s}\right]$

$\Delta$ : Filtering width [m]

$\Delta_{i}$ : Grid spacing in $\mathrm{X}$ direction [m]

$\Delta_{j}$ : Grid spacing in Y direction [m]

$\Delta_{k}$ : Grid spacing in $\mathrm{Z}$ direction [m]

$\Delta_{w}$ : Near-wall control volume length scale [m]

$\tau_{k}$ : Stress term of $k$ phase $\left[\mathrm{kg} \cdot \mathrm{m}^{-1} \cdot \mathrm{s}^{-2}\right]$

$\tau_{\omega}$ : Wall shear stress $\left[\mathrm{kg} \cdot \mathrm{m}^{-1} \cdot \mathrm{s}^{-2}\right]$

\section{REFERENCES}

1) Y. Miki and S. Takeuchi: ISIJ Int., 43 (2003), 1548.

2) H. Bai and B. G. Thomas: Metall. Mater. Trans., 32B (2001), 269.

3) K. H. Tacke: J. Iron Steel Res. Int., 18 (2011), 211.

4) K. Takatani, Y. Tanizawa and H. Mizukami: ISIJ Int., 41 (2001), 1252.

5) N. G. Deen, T. Solberg and B. H. Hjertager: Chem. Eng. Sci., 56 (2001), 6341.

6) L. F. Zhang, S. B. Yang, K. K. Cai, J. Y. Li, X. G. Wang and B. G. Thomas: Metall. Mater. Trans., 38B (2007), 63.

7) B. G. Thomas, A. Dennisov and H. Bai: 80th Steelmaking Conf. Proc., ISS, Warrendale, PA, (1997), 375.

8) Y. Miki, H. Ohno, Y. Kishimoto and S. Tanaka: Tetsu-to-Hagané, 97 (2011), 423.

9) B. K. Li, T. Okane and T. Umeda: Metall. Mater. Trans., 31B (2000), 1491.

10) B. K. Li and F. Tsukihashi: ISIJ Int., 45 (2005), 30.

11) B. G. Thomas, X. Huang and R. C. Suaaman: Metall. Mater. Trans., 25B (1994), 527.

12) D. Creech: Master's Thesis, University of Illinois at UrbanaChampaign, Urbana, IL, (1999).

13) H. Bai and B. G. Thomas: Metall. Mater. Trans., 32B (2001), 1143.

14) Z. Q. Liu, F. S. Qi, B. K. Li and F. Tsukihashi: J. Iron Steel Res. Int., 18 (2011), 243.

15) Q. Yuan, B. G. Thomas and S. P. Vanka: Metall. Mater. Trans., 48B (2012), 23.

16) B. K. Li, Z. Q. Liu, F. S. Qi, F. Wang and G. D. Xu: Acta Metall. Sin., 40 (2012), 88.

17) Z. D. Qian and Y. L. Wu: ISIJ Int., 44 (2004), 100.

18) C. Real, R. Miranda, C. Vilchis, M. Barron, L. Hoyos and J. Gonzalez: ISIJ Int., 46 (2006), 1183.

19) N. G. Deen, T. Solberg and B. H. Hjertager: Chem. Eng. Sci., 56 (2001), 6341.

20) M. T. Dhotre, B. Niceno and B. L. Smith: Chem. Eng. J., 136 (2008), 337.

21) H. A. Jakobsen, B. H. Sannaes, S. Grevskott and H. F. Svendsen: Ind. Eng. Chem. Res., 36 (1997), 4052.

22) Y. Sato and K. Sekiguchi: Int. J. Multiphas. Flow, 2 (1975), 79.

23) J. Smagorinsky: Monthly Weather Rev., 91 (1963), 99.

24) H. Werner and H. Wengle: 8th Symp. on Turbulent Shear Flows, Munich, Germany, (1991), 155.

25) D. A. Drew and R. T. Lahey: Int. J. Multiphas. Flow, 13 (1987), 113.

26) H. Bai and B. G. Thomas: Metall. Mater. Trans., 32B (2001), 253.

27) D. Gupta and A. K. Lahiri: Metall. Mater. Trans., 27B (1996), 757.

28) D. Gupta, S. Chakraborty and A. K. Lahiri: ISIJ Int., 37 (1997), 654. 